\title{
Comparative Efficacy and Mechanisms of a Single Session Behavioral Medicine Class among Patients with Chronic Pain Taking Prescription Opioids: Study Protocol for a Randomized Controlled Trial
}

\section{Abby L. Chen \\ Stanford University \\ Tyler Winslow \\ Stanford University \\ Sean C. Mackey \\ Stanford University \\ Beth D. Darnall \\ Stanford University}

Maisa Ziadni ( $\nabla$ mziadni@stanford.edu )

Stanford University School of Medicine https://orcid.org/0000-0001-8371-0646

\section{Study protocol}

Keywords: Chronic pain, prescription opioids, pain catastrophizing, cognitive-behavioral therapy, behavioral medicine, treatment

Posted Date: April 2nd, 2020

DOI: https://doi.org/10.21203/rs.3.rs-20549/v1

License: (c) (1) This work is licensed under a Creative Commons Attribution 4.0 International License. Read Full License

Version of Record: A version of this preprint was published at Trials on June 12th, 2020. See the published version at https://doi.org/10.1186/s13063-020-04415-x. 


\section{Abstract}

Background: Independent of pain intensity, pain-specific distress is highly predictive of pain treatment needs, including prescription opioids. Given the inherently distressing nature of chronic pain, there is a need to equip individuals with pain education and self-regulatory skills that are shown to improve adaptation and improve response to medical treatments. Brief, targeted behavioral medicine interventions may efficiently address the key individual factors, improve self-regulation in the context of pain, and reduce need for opioid therapy. This highlights the critical need for targeted, cost-effective interventions that efficiently address the key psychological factors that can amplify the need for opioids and increased risk for misuse. In this trial, the primary goal is to test the comparative efficacy of a single-session skillsbased pain management class to a health education active control group among patients with chronic pain who are taking opioids.

Methods: Our study is a randomized, double-blind clinical trial testing the superiority of our 2-hour singlesession skills-based pain management class against a 2-hour health education class. We will enroll 136 adult patients with mixed etiology chronic pain who are taking opioid prescription medication and randomize 1:1 to one of the two treatment arms. We hypothesize superiority for the skills-based pain class for pain control, self-regulation of pain-specific distress, and reduced opioid use measured by daily morphine equivalent. Team researchers masked to treatment assignment will assess outcomes up to 12 months post-treatment.

Discussion: This study aims to test the utility of a single-session, 2-hour skills-based pain management class to improve self-regulation of pain and reduce opioid use. Findings from our project have the potential to shift current research and clinical paradigms by testing a brief and scalable intervention that could reduce need for opioids and prevent misuse effectively, efficiently and economically. Further, elucidation of mechanisms of opioid use can facilitate refinement of more targeted future treatments.

\section{Background}

There is a critical need for reduced emphasis on high-risk pain treatments and better integration of behavioral medicine and self-management strategies to treat pain comprehensively by integrating a 'whole person' approach to pain care [1-3]. To date, the U.S. lacks scalable behavioral medicine for pain thereby underscoring the need for solutions that are accessible, low-cost, and low-burden. Evidencebased, skills-based behavioral medicine for pain has been shown to reduce pain-specific distress $[4,5]$, pain intensity [6], pain bothersomeness [7], improve response to pain treatments [8], and reduced opioid use among perioperative patients [9].

\section{Specific aims}

Our two specific aims and their corresponding hypotheses are outlined below. 
1. We will conduct a randomized controlled trial comparing the single-session skills-based pain management class to a single-session health education control (no actionable skills)

- Hypothesis 1a: The single-session skills-based pain management class will lead to greater reductions in opioid use compared to HE class.

- Hypothesis 1b: The single-session skills-based pain management class will lead to greater reductions in opioid misuse, pain-related distress (pain catastrophizing, depression, anxiety), and pain interference compared to the HE class.

2. To characterize the influence of daily pain catastrophizing on same-day and next-day opioid use

- Hypothesis 2a: Daily pain catastrophizing will predict same-day and next-day opioid use. Relationships between daily pain catastrophizing and same day and next day opioid use are reduced in the Empowered Relief (ER) class compared to HE.

- Exploratory Hypothesis 2b: Daily mean changes in pain catastrophizing (baseline to 3-month post-treatment) will predict reduction in opioid use, opioid misuse, and mean change in pain and function measures in the single-session skills-based pain class at 3, 6, and 12 months posttreatment.

\section{Methods/design}

\section{Overview}

We are conducting a randomized clinical trial in which individuals with a chronic pain condition who are currently taking prescription opioids are randomly assigned to one of two arms: a single-session skillsbased pain management class or a single-session health education class (active control; "Control") (Figs. 1 and 2). Participants will be followed for 12 months after treatment. Participants will be assessed via an online screening form, a phone screening, enrollment survey, pre-class survey, a two-week daily baseline period, 2-week daily follow-up period, and at 3, 6 and 12 months post-treatment. Team statisticians blinded to participant treatment assignment will assess outcomes three months after treatment, and after 6 and 12 months. The primary outcome is opioid use 3 months post-treatment. Secondary outcomes include reductions in pain-specific distress, pain interference and opioid misuse at 3 months.

The protocol for this trial has been approved by the Stanford Institutional Review Board (IRB). All participants will be required to give informed consent to a trained study team member prior to enrollment in the study.

\section{Study sample and setting}

Participants for this trial will be recruited through targeted emails and advertisements at Stanford's pain management clinics, in addition to the Stanford Systems Neuroscience and Pain Lab (SNAPL) database. 
Recruitment efforts will extend to social media marketing, and local advertisements in clinics and in the community. All advertisements will direct interested individuals to an online screening form that assesses for initial eligibility. The study will enroll 136 adults (age 18-80 years) with a chronic non-cancer pain condition, currently using prescription opioids $\geq 20$ morphine equivalent daily dose (MEDD) for $\geq 3$ months and who meet study criteria (Table 1). The sample size accounts for expected attrition. Eligibility will be assessed by the research staff.

\section{Inclusion and exclusion criteria}

Tables 1 and 2 list the inclusion and exclusion criteria, respectively, as well as the rationale for each criterion and the sources where each criterion will be assessed. Additionally, we require that the participants be willing and available to participate in the full treatment session to which they are assigned and able to respond to the daily measures (at baseline and follow-up) and post-treatment $(3,6$, and 12 months) follow-up questionnaires.

\section{Recruitment procedures}

Because the study intervention involves group treatment classes, we are recruiting participants in cohorts consisting of 7-12 participants per class cohort (minimum of 4 participants, maximum of 15 participants per cohort) for both treatment arms.

Interested individuals deemed initially eligible by the online screening will be further screened over the phone. Eligible individuals will then be invited to enroll in the study, and consented with a research staff over the phone, after which they provide an electronic signature to the consent form emailed to them. Participants are randomized following eligibility confirmation and informed consent procedures. Then participants complete the enrollment survey, which includes information related to their chronic pain, opioid use, non-opioid medication use, medical history, and psychosocial wellbeing. Additionally, measures of opioid misuse behaviors and severity, treatment expectancies and patient motivation factors will be administered.

\section{Randomization}

Enrolled participants will be randomized to one of two treatment arms: Empowered Relief (ER) and chronic pain health education class (HE). An automated program in REDCap will randomly assign a participant to a treatment arm when enrolled and will ensure blinded randomization, as well as equal numbers in both arms at the end of data collection. 


\section{Blinding}

Participants cannot and will not be blinded to the intervention they are randomized to. A clinical psychologist who will be trained in the intervention, blind to participants and has no involvement in data analysis will deliver the intervention. The study coordinator will be responsible for handling the randomization process through REDCap, but will remain blinded to the randomization scheme. All data given to the statistician will be blinded, except as required when reporting adverse events. All research data will be kept separate from identifiers and linked using a participant number. An alternative research team member will have access to the data and will be responsible for the data monitoring. Only the principal investigators will have access to the file linking names and participant numbers and the file will be stored in their locked offices. The team will have access to the final unidentified dataset.

\section{Study treatments}

Both study arms (ER and HE) consist of a single-session 2-hour group class. Participants will leave the ER class with home-based resources that facilitate ongoing self-regulation and pain self-management.

\section{Single-session skills-based pain class (Empowered Relief)}

Our group developed the single-session skills-based pain management class in 2013 with a goal of rapidly equipping patients with skills to self-regulate pain-specific distress. Pilot data revealed significantly reduced pain-specific distress - as indexed by reductions in pain catastrophizing- one month post-treatment regardless of comorbid depression and anxiety [4]. The single-session skills-based class ("Empowered Relief") is also the subject of $\mathrm{NIH}$-funded randomized controlled trial in chronic low back pain [5] with pain reduction as the primary outcome.

For this study, a clinical psychologist trained in delivering the 2-hour intervention will administer the class to groups of enrolled participants. Brief education on opioid reduction is included in the class, along with a one-page handout that summarizes key research findings. Materials are already in use in our existing projects [23]. The class is delivered by Powerpoint presentation and includes mind-body pain science, the importance of self-regulation in the context of pain and stress, and evidence-based skills that target painspecific distress and enhance pain control. Participants will be guided in developing their own selftreatment plan and acquiring the skills necessary to decrease pain and stress related physiological hyperarousal, and to enhance regulation of cognition and emotion within the context of pain. At the end of the class, participants will leave with: 1) a self-tailored personalized plan to target pain-specific distress; 2) a 20-minute guided relaxation response electronic app; and 3) a printed copy didactic class 
content. Cohort effects are expected to be minimal due to the single session nature of the class, the highly structured and manualized nature of the class, and minimal participant interaction.

\section{Health education class}

This is 2-hour class that will be delivered by a health educator using a PowerPoint presentation. The class will provide the participants with general health information related to exercise, nutrition and medication management. It includes information on managing flare-ups, working with healthcare professionals, evaluating treatments, and making informed decisions. This class is already in use in our $\mathrm{NIH}$-funded research $[5,24]$. This class serves as a control to the ER class, with matching factors, such as duration, structure, format, and location.

\section{Class sites}

All treatment sessions will occur at approved clinical or research sites within the Stanford University School of Medicine and Stanford HealthCare.

\section{Instructors}

For the ER treatment group, all instructors will be doctoral level clinical psychologists trained in the treatment of chronic pain. The HE class will be expert-led by experienced health educators or chronic pain professionals (e.g., chronic pain physician assistant).

\section{Training and monitoring of instructors}

ER instructors will be trained in the study protocol for their classes prior to administering treatment. Existing treatment manuals as well as highly structured and standardized class content will assure treatment fidelity. A research coordinator, serving as fidelity rater, will directly observe the first 3 classes of each treatment arm as well. Cohort effects are likely to be minimal, due to the single-session format and relatively minimal participant interaction.

\section{Measures}

Demographic data, chronic pain history, current and past opioid use, non-opioid medication, and current treatments will be collected at enrollment. The 17-item Current Opioid Misuse Measure [25] will be used to measure opioid misuse and change throughout the study. During screening, we will characterize the patient's opioid misuse behaviors using DSM-5 Opioid (DSO) [26], a Clinical Trials network NIDA- 
supported instrument. PROMIS measures will be used to assess Pain Interference, Physical Function, Depression, Anxiety, Anger, Sleep Disturbance, Fatigue, Social Isolation, and Global Health using short forms [27]. Our group has applied the NIH PROMIS measures in multiple nationally funded clinical pain treatment trials and other studies [28-30]. Pain Catastrophizing will be assessed using the Pain Catastrophizing Scale (subscales: rumination, magnification, and feelings of helplessness) [31]. Motivational Factors including desire, confidence, readiness and motivation to reduce their opioid use, will be assessed using the questions developed by Goesling and colleagues [32]. Lastly, treatment expectancies will be assessed using the Stanford Expectations of Treatment Scale (SETS) [33].

Baseline period: During screening and enrollment, patients will complete an online baseline assessment with demographics, as well as measures inquiring about pain condition(s) and characteristics, opioid use and misuse, pain catastrophizing and the PROMIS measures. Within one month of starting treatment, participants will also complete two weeks of daily surveys assessing daily levels of pain catastrophizing and daily opioid use.

Pre-treatment assessment: Three days pre-treatment, patients will complete an online pre-class survey assessing pain condition and characteristics, opioid use and misuse, pain catastrophizing, and the PROMIS measures. Participants will not be asked to repeat demographic information again, but these measures will be identical to those assessed at baseline.

Post-treatment assessment: Immediately post-treatment, patients will complete a brief questionnaire assessing patient satisfaction with the intervention on an 11-point Likert scale.

One-month post-treatment, participants will complete daily surveys assessing daily levels of pain catastrophizing and daily opioid use. For patients in the ER class, they will also complete measures inquiring about daily app and skill use for two weeks. At 3, 6, and 12 months post-treatment, all participants will complete a set of questionnaires identical to those administered pre-class. The primary study endpoint is three months post-treatment. App use will also be tracked by REDCap throughout the duration of the trial.

Participants may receive up $\$ 160$ for study completion.

Primary outcome measure. Our primary outcome measure is opioid use at three months post-treatment. We will convert opioid doses to morphine equivalent daily dose (MEDD). We will calculate a withinsubject difference from baseline to determine if reduction in MEDD use occurred. 
Secondary outcome measures. Our secondary outcome is pain-specific distress as indexed by pain catastrophizing scale scores at 3-months post treatment. Pain catastrophizing will be assessed using the Pain Catastrophizing Scale (PCS) and a total score will be generated using the sum of the 13-items of the PCS. The PCS consists of three subscales (helplessness, magnification, rumination) and has good psychometrics [31].

Tertiary outcome measures. NIH PROMIS measures [34] will be administered to assess Anxiety, Depression, Anger, Fatigue, Pain Interference, Physical Function, Global Health, and Sleep Disturbance. These measures have been successfully applied to pain research [13, 35-37].

Daily measures. All participants will complete daily measures of opioid medication use, pain intensity, and 3-item daily PCS during two 2-week time periods: at baseline (up to one month prior to the class), and at follow-up (one month post-class). Daily skills use will also be assessed for 2-weeks during the follow-up time-period only for the ER treatment arm. These include a 4-item questionnaire measuring frequency of use of cognitive, behavioral, or psychophysiological techniques over the past 24 hours from 0 times to $5+$ times.

\section{Data collection, quality control and confidentiality}

The online assessments completed by participants will be gathered securely in a REDCap database. Though we do not expect any questionnaires to be collected on paper, if unforeseen circumstances require this to occur, they will be stored as source data and a member of the study team will manually enter the responses into the REDCap database. Additionally, members of the team will be trained to use and complete Case Report Forms (CRFs), how to review them for completeness, as well as how to maintain participant confidentiality. Patient flow will be reported according to the CONSORT (Consolidated Standards of Reporting Trials) guidelines [39].

\section{Protection of human participants and assessment of safety:}

\section{Protection of human participants}

The Stanford University Institutional Review Board (IRB) approved this study. 


\section{Safety Monitoring}

This trial will be monitored for safety by an independent Data and Safety Monitoring Board (DSMB) composed of a biostatistician and a clinical psychologists with knowledge in treatment of chronic pain conditions. A chairperson of the board will also be appointed who is an individual with expertise in treatment outcome research methodology and who has worked as a consultant on other clinical trial studies and DSMB boards. These members will have no other involvement in the study and will serve as independent reviewers of the DSMB. They will convene twice a year or as per needed basis. Members of the DSMB will make relevant safety decisions regarding reported participant cases. The DSMB report will be sent to NIH within 2 weeks of the meeting, twice a year. The report will be sent to the Stanford IRB after meetings have been held for the year, and prior to the continuing renewal.

\section{Adverse experiences}

The treatments in this study are not anticipated to pose any risks to participants. However, a study coordinator will review enrolled patient records weekly to monitor for adverse events. In the case of an adverse event, an Adverse Event Case Report Form will be completed. These will be discussed in monthly team meetings and reported to the DSMB and IRB annually. Serious adverse events will be reported to the Stanford IRB and NIH. The DSMB and PI will evaluate all serious adverse events within 24 hours after the study team becomes aware of the incident. All study-related adverse events will be included in the annual report to the $\mathrm{NIH}$, and serious adverse events will be reported within 2 weeks.

\section{Stopping rules}

The trial will be stopped if (1) either treatment intervention is associated with adverse effects that calls into question the safety of the intervention, (2) difficulty in study recruitment or retention that will impact the ability to evaluable study endpoints, (3) new information becomes available during the trial that indicates the need to stop the trial, or (4) other unforeseen situations that would warrant stopping the study.

\section{Statistical issues}

\section{Sample size and detectable differences}

We chose our sample size to ensure adequate power to detect treatment effects on the primary outcome (i.e. opioid use) and to investigate pain catastrophizing reduction as a mediator between the two groups (ER and HE). The project will enroll 136 participants (ages 18-80 years) with diagnosis of chronic noncancer pain (>3 months in duration) and currently using prescription opioids. 
To compare the main effect of single-session skills-based pain management class on opioid use against the HE control condition, we will plan to enroll 136 participants and have 116 completers (58 per group). The proposed sample size accounts for $15 \%$ attrition in each treatment arm. This is lower than the current attrition rate seen in pain-CBT literature of $18-25 \%[40,41]$, but we believe our less burdensome singlesession groups will lead to lower rates. We hope to achieve $80 \%$ power to detect medium-large treatment effects on the primary outcome (i.e. opioid use).

To examine reduction of PC as a treatment mediator, we will use bias-corrected bootstapping. Previous data show a large effect of PC treatment on PCS scores ( $d=0.85 \sim 1.15)$ [4], and others show a mediumsize effect of PCS scores on opioid use $(d=0.4)$ [32]. When using bias-corrected bootstrap for mediation analysis, a total of 115 subjects are required for a large and medium effect associations with a mediator variable (PCS score), $80 \%$ power and $a=0.05$ [42].

\section{Statistical analyses}

\section{Primary analyses}

We will use an intent-to-treat approach in all analyses (i.e. the assessment of individuals will be analyzed by randomized group, regardless of participation in any classes). By doing so, we protect against any confounding that arises as a result of subject dropout.

The main effect of the single-session skills-based pain management class on opioid use will be compared against the HE control class using a 2-samples t-test. Clinically minimal reduction is defined as $>15 \%$ reduction in opioid use [43] in MEDD, the recommended unit of measurement in studies of opioid use [38]. We will compare the rate of participants who reach clinically minimal reduction between the two groups. We will quantify absolute opioid reduction in addition to percent change reduction within subject and between classes. Finally, we will quantify percent achieving each group threshold for importance of change $(15 \%, 30 \%$, and $50 \%$ as minimally, moderately and substantially important change scores, respectively).

To test the hypothesis that the ER class will have greater reductions in pain-related distress, pain interference, and opioid misuse compared to the HE class, our endpoint is opioid misuse, pain catastrophizing, and pain interference at 3 months, and its within-subject difference from baseline is calculated. The mean difference in the ER group will be compared against the HE arm using the twosample t-test. We will also compare the proportion of success rate, defined as $\geq 30 \%$ reduction in pain catastrophizing and pain interference for clinical significant treatment response [44] and a reduction to 13 as measured by the COMM (Current Opioid Misuse Measure). This number if the validated cut-point on COMM [45]. 


\section{Secondary objectives}

To test the hypothesis that Daily PC predicts same-day and next-day opioid use, a mixed effects model will be used to study this association. The subject specific random effects will be used to account for subject-level (level 2) effects, in particular the effect of the intervention as well as daily level variations in PC.

Mixed effects regression will be used to study the association between the 1-month change in daily PC mean and the post-treatment opioid use, the outcome of interest. The regression will adjust for baseline opioid use and other confounding factors. Same analyses will be conducted with opioid misuse, pain intensity and pain interference as outcomes.

\section{Discussion}

In this trial, we will seek to determine whether a single-session skills-based behavioral pain medicine class is an effective treatment option for persons with chronic pain who are taking prescription opioids. The study will identify a proportion of patients who achieve a meaningful reduction in opioid use in response to this brief intervention. This will facilitate the future application of a refined version of the class across a variety of settings-such as in primary care or in pre-surgical populations. The study will also elucidate the mechanisms that change opioid use with and without targeted treatment. This information will not only reveal importanat mechanisms at play, but will also allow us to better characterize responders and non-responders to treatment, which will facilitate the development of more tailored and targeted interventions in the future.

\section{Trial Status}

NCT03950791 was registered on 05/19. Recruitment began in 09/19. Expected data when recruitment will be completed is $05 / 2023$. IRB (protocol \#48784) was initially approved on $12 / 18 / 2018$.

\section{Declarations}

Ethics approval and consent to participate

Study was approved by Stanford's Institutional Review Board (protocol \# 48784) 3000 El-Camino Real, Five Palo Alto Square, $4^{\text {th }}$ Floor, Palo Alto, CA 94306. An informed consent will be obtained from all participants in the study.

Consent for publication

Not applicable 
Availability of data and material

Data will be available on clinicaltrials.gov (NCT03950791)

Competing Interests: All authors declare that they have no competing interests.

Funding: NIH K23DA047473 (MSZ). The awarding NIDA review board contributed to the design of the study, selection of instruments, and methods of data collection.

Author contributions. MSZ, BDD, and SCM conceived of the trial. BDD created the single-session skillsbased pain management intervention. MSZ, BDD, TW, and SCM refined the protocol and selected measures. MZ and BDD developed plans for the statistical analyses. MSZ, AC, and TW drafted the manuscript. All authors read and approved the final manuscript.

Acknowledgements: Thanks to National Institute of Health for providing funding (NIH K23DA047473, K24DA029262, T32DA035165 and R01AT008561). The design of this clinical trial was approved by the NIDA Office of Clinical and Regulatory Affairs.

\section{References}

1. Institute of Medicine of the National Academies. Institute of Medicine: Relieving Pain in America. 2011.

2. National Institutes of Health. National Pain Strategy: A Comprehensive Population Health-Level Strategy for Pain. 2016.

3. U.S. Department of Health \& Human Services. Report on Pain Management Best Practices: Updates, Gaps, Inconsistencies, and Recommendations. 2019.

4. Darnall BD, Sturgeon JA, Kao MC, Hah JM, Mackey SC. From catastrophizing to recovery: a pilot study of a single-session treatment for pain catastrophizing. J Pain Res. 2014;7:219.

5. Darnall BD, Ziadni MS, Roy A, Kao MC, Sturgeon JA, Cook KF, et al. Comparative efficacy and mechanisms of a single-session pain psychology class in chronic low back pain: Study protocol for a randomized controlled trial. Trials. 2018;19(1):165.

6. Naylor MR, Seminowicz DA, Somers TJ, Keefe FJ. Pain Imaging. In: Moore RJ, editor. Handbook of Pain and Palliative Care: Biobehavioral Approaches for the Life Course. New York: 2013. p. 439-467.

7. Cherkin DC, Sherman KJ, Balderson BH, Cook AJ, Anderson ML, Hawkes RJ, et al. Effect of Mindfulness-Based Stress Reduction vs Cognitive Behavioral Therapy or Usual Care on Back Pain and Functional Limitations in Adults With Chronic Low Back Pain: A Randomized Clinical Trial. JAMA. 2016;315(12):240-1249.

8. Burns JW, Gleen B, Bruehl S, Harden RN, Lofland K. Cognitive factors influence outcome following multidisciplinary chronic pain treatment: a replication and extension of a cross-lagged panel analysis. Behav Res Ther. 2003;41(10):1163-1182. 
9. Darnall BD, Ziadni MS, Krishnamurthy P, Flood P, Heathcote LC, Mackey IG, et al."My Surgical

Success": Effect of a Digital Behavioral Pain Medicine Intervention on Time to Opioid Cessation After Breast Cancer Surgery-A Pilot Randomized Controlled Clinical Trial. Pain Med. 2019;20(11): 22282237.

10. (NPSTF), N.P.S.T.F. National Pain Strategy - A Comprehensive Population Health-Level Strategy for Pain, National Institutes of Health, Editor. 2015.

11. Von Korff M, Scher Al, Helmick C, Carter-Pokras O, Dodick DW, Goulet J, et al. United States National Pain Strategy for Population Research: Concepts, Definitions, and Pilot Data. J Pain. 2016;17(10):1068-1080.

12. Boudreau D, Von Korff M, Rutter CM, Saunders K, Ray GT, Sullivan MD, et al. Trends in long-term opioid therapy for chronic non-cancer pain. Pharmacoepidemiol Drug Saf. 2009;18(12):1166-1175.

13. Mojtabai R. National trends in long-term use of prescription opioids. Pharmacoepidemiol Drug Saf. 2017;27(5):1-9.

14. Weisner CM, Campbell Cl, Ray GT, Saunders K, Merrill JO, Banta-Green C. Trends in prescribed opioid therapy for non-cancer pain for individuals with prior substance use disorders. Pain. 2009;145(3): 287-293.

15. Warner M, Chen LH, Makuc DM. Increase in fatal poisonings involving opioid analgesics in the United States, 1999-2006. NCHS Data Brief. 2009;(22):1-8.

16. Naylor MR, Naud S, Keefe FJ, Helzer JE. Therapeutic Interactive Voice Response (TIVR) to reduce analgesic medication use for chronic pain management. J Pain. 2010;11(12):1410-1419.

17. Jamison RN, Ross EL, Michna E, Chen LQ, Holcomb C, Wasan AD. Substance misuse treatment for high-risk chronic pain patients on opioid therapy: a randomized trial. Pain. 2010;150(3):390-400.

18. Guarino H, Fong C, Marsch LA, Acosta MC, Syckes C, Moore SK, et al. Web-based cognitive behavior therapy for chronic pain patients with aberrant drug-related behavior: Outcomes from a randomized controlled trial. Pain Med. 2018;19(12):2423-2437.

19. Thorn BE, Boothby JL, Sulllivan MJ. Targeted treatment of catastrophizing for the management of chronic pain. Cogn Behav Pract. 2002;9(2):127-138.

20. Rini C, Williams DA, Broderick JE, Keefe FJ. Meeting them where they are: using the internet to deliver behavioral medicine interventions for pain. Transl Behav Med. 2012;2(1):82-92.

21. Simon LS. Relieving pain in America: A blueprint for transforming prevention, care, education, and research. J Pain Palliat Care Pharmother. 2012;26(2):97-198.

22. Williams DA. Web-based behavioral interventions for the management of chronic pain. Curr Rheumatol Rep. 2011;13(6):543-9.

23. Darnall BD, Ziadni MS, Stieg RL, Mackey IG, Kao MC, Flood P. Patient-Centered Prescription Opioid Tapering in Community Outpatients With Chronic Pain. Jama Intern Med. 2018;178(5):707-708.

24. Sharifzadeh Y, Kao MC, Sturgeon JA, Rico TJ, Mackey S, Darnall BD. Pain catastrophizing moderates relationships between pain intensity and opioid prescription nonlinear sex differences revealed using 
a learning health system. Anesthesiology. 2017;127(1):136-146.

25. Butler SF, Budman SH, Fernandez KC, Houle B, Benoit C, Katz N. Development and validation of the current opioid misuse measure. Pain. 2007;130(1-2):144-156.

26. National Institute on Drug Abuse. DSM 5 - Opioids (DSO). Bethesda(MD): NIDA Clinical Trials Network. 2017.

27. Cella D, Riley W, Stone A, Rothrock N, Reeve B, Yount S, et al. The Patient-Reported Outcomes Measurement Information System (PROMIS) developed and tested its first wave of adult selfreported health outcome item banks: 2005-2008. J Clin Epidemiol. 2010;63(11):1179-1194.

28. Darnall BD, Sturgeon JA, Cook KF, Taub CJ, Roy A, Burns JW, et al. Development and Validation of a Daily Pain Catastrophizing Scale. J Pain. 2017;18(9):1139-1149.

29. Sturgeon JA, Hah JM, Sharifzadeh Y, Middleton SK, Rico T, Johnson KA, et al. Predictors of Daily Pain Medication Use in Individuals with Recurrent Back Pain. Int J Behav Med. 2018; 25(2):252-258.

30. Ziadni MS, Sturgeon JA, Darnall BD. The relationship between negative metacognitive thoughts, pain catastrophizing and adjustment to chronic pain. Eur J Pain. 2018;22(4):756-762.

31. Sullivan MJ, Bishop SR, Pivik J. The pain catastrophizing scale: development and validation. Psychol Assess. 1995;7(4):524.

32. Goesling J, Moser SE, Lin LA, Hassett AL, Wasserman RA, Brummet CM. Discrepancies between perceived benefit of opioids and self-reported patient outcomes. Pain Med. 2016;19(2):297-306.

33. Younger J, Gandhi V, Hubbard E, Mackey S. Development of the Stanford Expectations of Treatment Scale (SETS): A tool for measuring patient outcome expectancy in clinical trials. Clin Trials. 2012;9(6):767-776.

34. Linton SJ. Do psychological factors increase the risk for back pain in the general population in both a cross-sectional and prospective analysis? Eur J Pain. 2005;9(4):355-361.

35. Edlund MJ, Martin BC, Fan MY, Devries A, Braden JB, Sullivan MD. Risks for opioid abuse and dependence among recipients of chronic opioid therapy: results from the TROUP study. Drug Alcohol Depend. 2010;112(1):90-98.

36. Compton WM, Volkow ND. Major increases in opioid analgesic abuse in the United States: concerns and strategies. Drug Alcohol Depend. 2006;81(2):103-107.

37. Boscarino JA, Rukstalis M, Hoffman SN, Han JJ, Erlich PM, Gerhard GS, et al. Risk factors for drug dependence among out-patients on opioid therapy in a large US health-care system. Addiction. 2010;105(10):1776-1782

38. Svendsen K, Borchgrevink P, Fredheim O, Hamunen K, Mellbye A, Dale O. Choosing the unit of measurement counts: the use of oral morphine equivalents in studies of opioid consumption is a useful addition to defined daily doses. Palliat Med. 2011;25(7):725-732.

39. Boutron I, Moher D, Altman DG, Schulz KF, Ravaud P. Extending the CONSORT statement to randomized trials of nonpharmacologic treatment: explanation and elaboration. Ann Intern Med. 2008;148(4):295-309. 
40. Thorn BE, Pence LB, Ward LC, Kilgo G, Clements KL, Cross TH, et al. A randomized clinical trial of targeted cognitive behavioral treatment to reduce catastrophizing in chronic headache sufferers. J Pain. 2007;8(12):938-949.

41. Glombiewski JA, Hartwich-Tersek J, Rief W. Attrition in Cognitive-behavioral Treatment of Chronic Back Pain. Clin J Pain. 2010;26(7):593-601.

42. Fritz MS, Mackinnon DP. Required sample size to detect the mediated effect. Pyschol Sci. 2007;18(3):233-239.

43. Farrar JT, Portenoy RK, Berlin JA, Kinman JL, Strom BL. Defining the clinically important difference in pain outcome measures. Pain. 2000;88(3):287-294.

44. Dworkin RH, Turk DC, McDermott MP, Peirce-Sadner S, Burke LB, Cowan P, et al. Interpreting the clinical importance of group differences in chronic pain clinical trials: IMMPACT recommendations. Pain. 2009;146(3):238-44.

45. Meltzer EC, Rybin D, Saitz R, Samet JH, Schwartz SL, Butler SF, et al. Identifying prescription opioid use disorder in primary care: Diagnostic characteristics of the Current Opioid Misuse Measure (COMM). Pain. 2011;152(2):397-402.

\section{Tables}

Table 1 Inclusion criteria

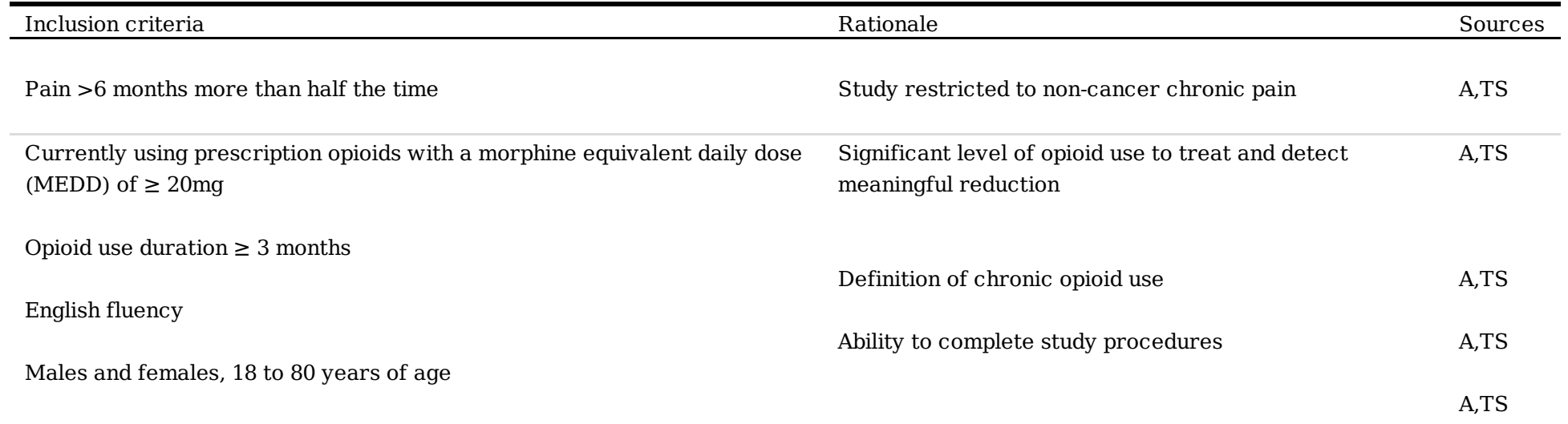

A, Automated data gathered from REDCap Surveys; TS, Telephone Screening

Table 2 Exclusion criteria 
Open litigation regarding a medical condition

Inability to provide informed consent and complete study procedures

Not able to complete study procedures

A,TS, E

Active participation in CBT-based treatment

Possible bias due to current exposure to treatment groups

Active suicidality

A, Automated data gathered from REDCap Surveys; TS, Telephone Screening; E, enrollment survey

Table 3 Baseline and follow-up measures

Figures 

treatments, pain diagnosis, current medical care related to chronic pain, psychological conditions

Non-Opioid A measure to identify which non-opioid medications participants are Medication Use taking in conjunction with their dose

Opioid Use Measure to assess misuse of prescribed and non-prescribed opioid Disorder (OUD) medications along with symptoms and outcomes

[26]

Pain Measures the individual's pain intensity at its worst and average

Characteristics intensity for the past 7 days. Also assesses current pain intensity. All of which use a 10-point scale from 'no pain' to 'very severe'
$\mathrm{X}$

Opioid Use [38] Current use of prescription opioids $\geq 20 \mathrm{mg}$ morphine equivalent daily dose (MEDD) for $\geq 3$ months

$\mathrm{x}$

$\mathrm{x}$ $\mathrm{x}$

$\mathrm{x}$

Current Opioid 17-item measure assessing risk for aberrant medication related Misuse

Measure

(COMM) [25] behavior among persons with chronic pain who are prescribed opioids for pain

Pain 13-item scale assesses severity of trait pain catastrophizing tendencies

Catastrophizing on a 5-point scale ( $0=$ "not at all"; 4 = "all the time"); sum scores

Scale range from 0-52.The PCS has 3 factors (helplessness, magnification,

(PCS) [31] rumination) and has good psychometrics [31]. Higher score reflects more catastrophizing

$\mathrm{x}$

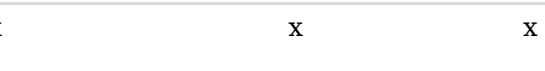

NIH PROMIS measures will be used to assess multiple variables of interest, including Pain Intensity, Pain Interference, Pain Behavior, Physical Function,

PROMIS Depression, Anxiety, Sleep Disturbance, Sleep Interference, Anger,

Measures [27] and Fatigue. Short form 8-item measures will be used to minimize participant burden.

Measurement Brief Measure Description

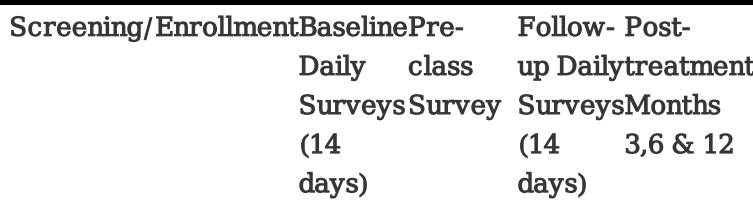

Stanford Stanford Expectations of Treatment Scale, a 6-item tool our group

Expectancy of developed and validated at SNAPL, will be used to assess participant

expectations of treatment 


\section{Factors [32] desire to decrease opioid use and their confidence in handling the pain} with said opioid decrease.

Daily

Measures\Skills

Use

A measure to identify opioid medications that individuals are taking daily in conjunction with their dose.

Daily Opioid

Use

Daily Pain The 3-item Daily PCS will be administered each day for 2 weeks prior

$\mathrm{x}$

$\mathrm{X}$ Catastrophizing to and post-treatment.

Scale

Skills Use for 4-item measure assessing frequency of self-regulatory skills learned in $\mathrm{x}$ Empowered class over the past month from not all to several times per day (5+)

Relief Class

[5]

Guided

Relaxation

Resource for

Empowered

Relief Class

[5]
Audio file resource that ER class participants download and can listen to any time.
Postclass 

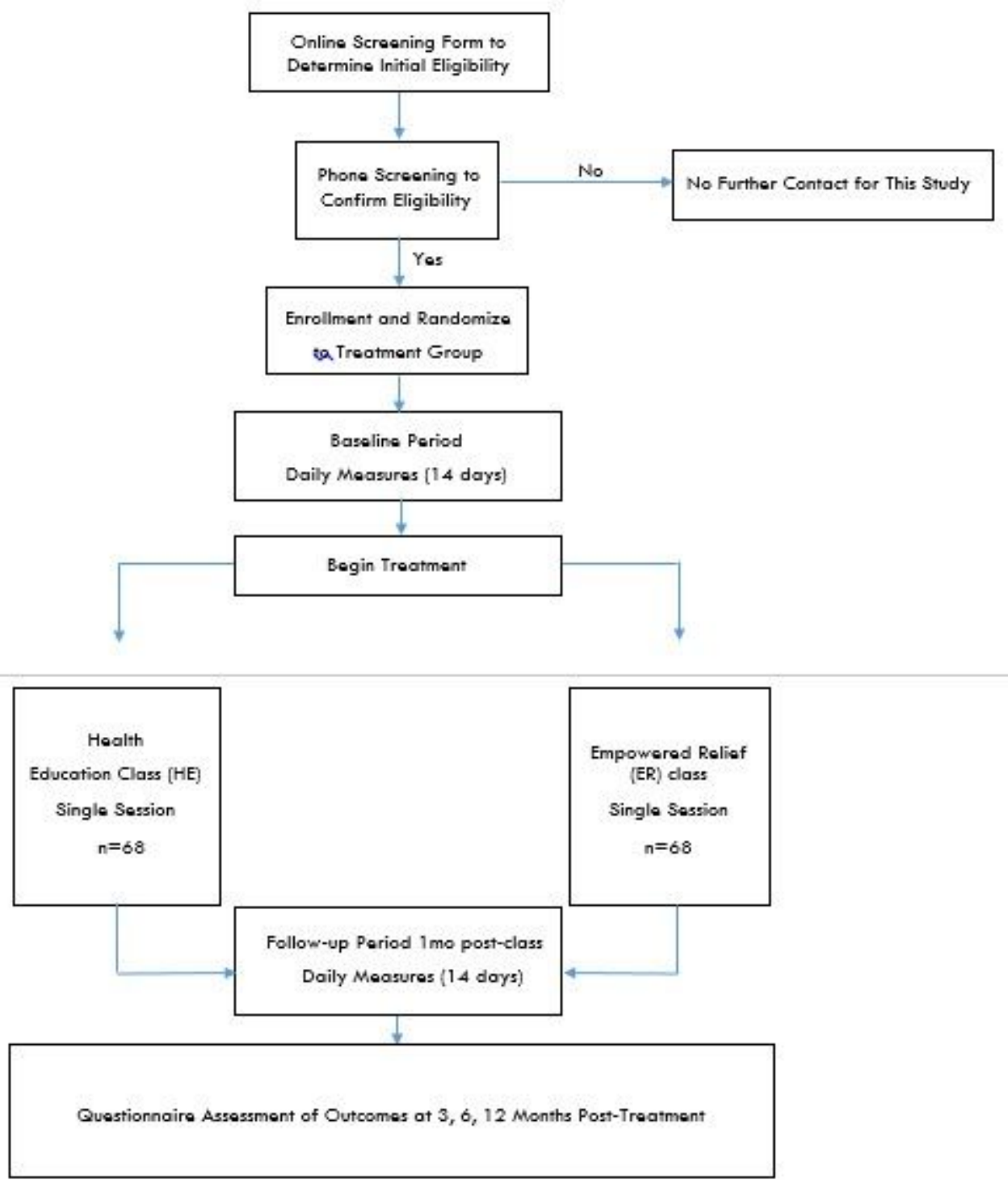

\section{Figure 1}

Flowchart of the trial protocol 


\begin{tabular}{|c|c|c|c|c|c|c|c|c|c|}
\hline \multicolumn{10}{|c|}{ STUDY PERIOD } \\
\hline & & \multirow{2}{*}{$\begin{array}{l}\text { Baseline } \\
\text { Baseline } \\
\text { Dailies } \\
14 \text { days }\end{array}$} & \multicolumn{3}{|c|}{$\begin{array}{c}\text { Interventions } \\
\text { Single-Session Skills-Bosed } \\
\text { class } \\
\text { or Health Education }\end{array}$} & \multicolumn{4}{|c|}{ Follow-up Assessments } \\
\hline & $\begin{array}{l}\text { Screening/f } \\
\text { Randomization }\end{array}$ & & $\begin{array}{l}\text { Pre- } \\
\text { Class } \\
\text { Survey }\end{array}$ & $\begin{array}{l}\text { Class } \\
\text { (ER, HE) }\end{array}$ & $\begin{array}{l}\text { Post- } \\
\text { Class } \\
\text { Survey }\end{array}$ & $\begin{array}{l}\text { Follow } \\
\text {-up } \\
\text { Dailies } \\
14 \\
\text { days }\end{array}$ & $\begin{array}{l}3 \\
\text { Month }\end{array}$ & $\begin{array}{l}6 \\
\text { Month }\end{array}$ & $\begin{array}{l}12 \\
\text { Month }\end{array}$ \\
\hline TLMEPOINT & th & $-t_{2}^{2}$ & $-t$ & \multicolumn{2}{|c|}{ to } & $\begin{array}{c}n \\
(200 \cos x)\end{array}$ & 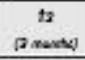 & $t_{(2 \pi)}^{t a n}$ & 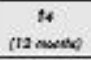 \\
\hline ENROLLMENT: & & & & & & & & & \\
\hline Eligibility screan & $x$ & & & & & & & & \\
\hline Allocation & $x$ & & & & & & & & \\
\hline Informed consent & $x$ & & & & & & & & \\
\hline $\begin{array}{l}\text { Assessments listed in } \\
\text { table } 3\end{array}$ & $\mathrm{x}$ & & & & & & & & \\
\hline $\begin{array}{l}\text { Daily Diary } \\
\text { Questions }\end{array}$ & & $x--x$ & & & & & & & \\
\hline INTERVENTION: & & & & & & & & & \\
\hline $\begin{array}{l}\text { Assessmonts listed in } \\
\text { table } 3\end{array}$ & & & $x$ & & & & & & \\
\hline [Empowored Relief] & & & & $\begin{array}{l}\text { Single } \\
\text { Session }\end{array}$ & & & & & \\
\hline [Health Education] & & & & $\begin{array}{l}\text { Single } \\
\text { Session }\end{array}$ & & & & & \\
\hline $\begin{array}{l}\text { Satisfaction with } \\
\text { Treatment Scale }\end{array}$ & & & & & $x$ & & & & \\
\hline $\begin{array}{l}\text { Daily Diary } \\
\text { Questions }\end{array}$ & & & & & & $x--x$ & & & \\
\hline ASSESSMENTS: & & & & & & & & & \\
\hline $\begin{array}{l}\text { Assessmonts listed in } \\
\text { table } 3\end{array}$ & & & & & & & $\mathrm{x}$ & $\mathrm{x}$ & $\mathrm{x}$ \\
\hline
\end{tabular}

Figure 2

The schedule of enrollment, interventions, and assessments

\section{Supplementary Files}

This is a list of supplementary files associated with this preprint. Click to download.

- SPIRITchecklist.doc 